\title{
Antonio Catiforo, Vita di Pietro and its Romanian Versions in the $18^{\text {th }}$ Century
}

\section{Dr. Eugenia Dima}

\author{
Faculty of Letters \\ "Alexandru loan Cuza" University of lasi, Romania
}

\author{
Doi:10.5901/jesr.2013.v3n7p368
}

\begin{abstract}
An interesting example of the way in which authors and translators got informed in the 18th century is represented by the book Vita di Pietro by Antonio Catiforo, printed in Venice in 1736. The author, of Greek origin, wrote his work in Italian and presented the life, activity and personality of the Russian Tsar Peter I the Great, mentioning in the foreword English, French and German sources. Cornaro's work, in two volumes (volume I, 4 parts and volume II, 2 parts), was almost immediately translated into Greek by Alexandros Kanghellarios and published in 1737 in Venice as well.In 1749, volume I of the Greek version was translated into Romanian by the grand nobleman of Wallachia Matei Fărcăşanu. His translation, not printed, was however known and circulated in several manuscript copies. The Greek version was entirely translated into Romanian in Moldavia by the clergyman Cozma Vlahul from the lasi Metropolitanate. His work is preserved in manuscript copies. A special situation is represented by another manuscript version from Brasov (Transylvania), which includes volume I, while volume II (part 5), though it is mentioned to be part of the same work, is entirely different from Cornaro's work, including a compilation of Romanian chronicles and information regarding the situation of the Swedish and Russian troupes after the Russian-Swedish war. At the middle of the 18th century there was a Russian version of Cornaro's two volumes.

Such presentation will illustrate the regime of the writings in the 18th century at European level.
\end{abstract}

\section{Antonio Catiforo, Vita di Pietro e le sue Versioni Rumene dell'Ottocento}

La rivoluzione intellettuale del Settecento stimolò l'investigazione della realtà e contemporaneamente gli intellettuali di tutta Europa parteciparono direttamente alla ricerca scientifica oppure cercarono di assimilarne i risultati, coscienti dell'importanza dei dati provenienti dalla meccanica e dalla matematica, dall'astronomia o dalla biologia, e individuarono nuovi settori di attività per la ragione umana. Verso la fine dell'Età dei Lumi, la scienza cominciò ad acquisire un'importanza decisiva nella vita intellettuale delle società europee e divenne un modo di pensare, cancellando i pregiudizi e determinandone il progresso. La superstizione e la paura fecero spazio alle scienze intente allo studio della natura, della vita e del cosmo. L'apertura verso una nuova forma di universalità non fu facile nelle società dell'Europa Occidentale, dove il mutamento dei concetti si accellerò bruscamente sotto l'impulso di una nuova mentalità, mentre i rinnovamenti del periodo illuminista contribuirono all'amplificazione dello choc provocato dalla istituzione di nuovi rapporti culturali.

Per le società dell'Europa del Sud - Est, il progresso delle civiltà occidentali costituì un importante riferimento, con una grande forza di attrazione. Andando da ovest a est, la struttura dei livelli culturali si presenta in movimento, ma sempre più lenta man mano che ci si avvicina al polo sud-estico. L'assimilazione delle culture occidentali si fece senza scosse violenti: gli intellettuali non rigettarono le conquiste del passato, tanto da prevenire una meccanica integrazione nel mondo europeo dell'ovest, che avrebbe portato all'uniformizzazione. L'interculturalità in questo periodo, consistente nell'interesse per la cultura, la storia o la geografia di altri paesi, fu anch'essa un effetto dell'lluminismo. Gli studiosi desiderarono conoscere sempre più cose nuove, nuovi orizzonti e appropriarsi del progresso di altre nazioni, che riguardava soprattutto la scienza, la cultura e la civiltà.

Un caso interessante per il modo in cui si informavano gli autori, ma anche i traduttori, è rappresentato dal lavoro Vita di Pietro il Grande Imperador della Russia di Antonio Catiforo, uscito a Venezia nel 1736. Sulla vita e sull'attività di Antonio Catiforo si hanno varie informazioni grazie all'eccellente lavoro di Margherita Losacco, dal quale riprendiamo alcune informazioni. Catiforo nacque nell'isola di Zante, territorio veneziano già da due secoli, di una famiglia borghese di origine ateniese, probabilmente intorno al 1685 . Vi restò fino al 1702, quando andò in Italia, a completare gli studi a Roma e a Venezia. Seguì il Collegio Greco di Roma, periodo in cui si occupò della supervisione della biblioteca del colleggio e ricoprì anche alcuni incarichi didattici. Si ritirò dal colleggio prima di essere ordinato e si stabilì a Venezia, dove entrò in contatto con la comunita greca, diventando diacono. Tornò a Zante nel 1710, dove diventò ance vescovo di 
Cefalonia e Zante, ma nel 1732 fu di nuovo alla volta di Venezia. Affascinato dalla personalità dello tzar Pietro I, ma anche nella speranza di un eventuale sostegno dello tzar per i greci ortodossi, si imbarcò per la Russia ma, a seguito del naufragio della nave, si fermò in Ollanda. Ritornò a Venezia attraverso il Belgio, la Francia e la Germania e vi rimase fino alla metà degli anni Cinquanta, svolgendo attività di insegnante e predicatore. Finalmente, ritornò a Zante dove trascorse l'ultimo periodo della sua vita, fino alla morte, nel 1763 (Losacco, 2001, pp. 39-154).

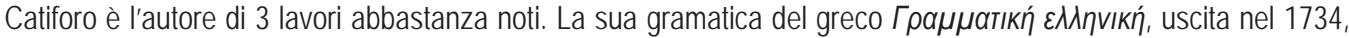
conobbe una seconda edizione nel 1737 e ben 4 edizioni postume. II lavoro è di carattere ampiamente compilativo,

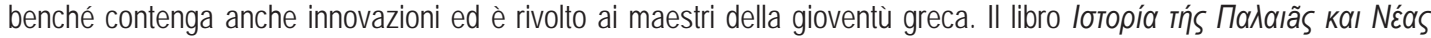

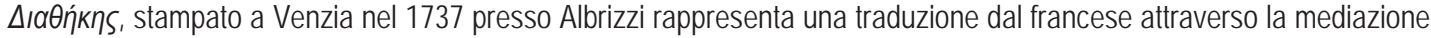
italiana.

II volume di cui ci occuperemo in seguito, è la Vita di Pietro il Grande, imperador della Russia; estratta da varie memorie pubblicate in Francia, e in Olanda, Venezia, appresso Francesco Pitteri, 1736. In questa prima edizione il nome dell'autore non figura, come non figura neanche nella ristampa del 1737. Solo nel 1739, la seconda edizione, riveduta ed accresciuta dall'autore, reca il nome di Catiforo. II libro ebbe tre successive edizioni 1748, 1781, 1792. L'autore di origine greca, che scrisse il suo lavoro in italiano, professa il proprio interesse per lo zar russo Pietro I il Grande e presenta nel suo libro la vita, l'attività e la personalità dello zar, menzionando nella premessa anche le fonti inglesi, francesi 0 tedesche che aveva utilizzato per il suo scritto. Queste sono: B. Iwan Nestesuranoi, Friedrich Christian Weber e John Perry.

B. Iwan Nestesuranoi (pseudonimo di Jean Rousset de Missy) fu uno storico francese della prima metà del Settecento. Anche se non si crede che abbia visitato la Russia, egli utilizzò informazioni fornite dai viaggiatori contemporanei 0 altre fonti per compilare una vita di Pietro il Grande (Mémoires du règne de Pierre le Grand, empereur de Russie, Amsterdam, Wetstein e Smith, 1728 - 1730).

Friedrich Christian Weber (n.s. - 1739) fu ambasciatore presso la corte di Pietro il Grande in Russia. I dati biografici, pochi, sono stati estratti dalla sua corrispondenza diplomatica. II suo lavoro, Das Veränderte Russland, uscì in tre volumi nel 1721, nel 1739 e nel 1740.

John Perry (1670-1733) entrò nella Marina Regale e, dopo una vita avventurosa nella sua carriera di capitano di nave, si specializzò in ingegneria idraulica e fu inviato come specialista e ambasciatore in Russia, che lasciò nel 1712. Ritornato in Inghilterra, costrui vari frangiflutti e dighe. Nel 1716 scrisse il volume The State of Russia, under the Present Czar /Peter the Great/, stampato a Londra.

II libro di Catiforo, che comprende 2 volumi (volume I, 4 libri e volume II, 2 libri), fu quasi subito tradotto in greco da

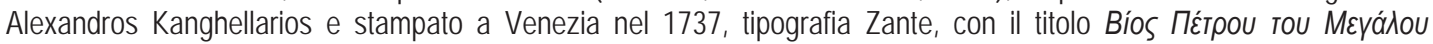

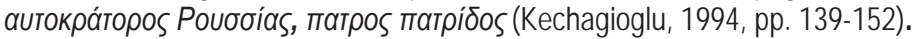

Nella lingua rumena esistono 3 versioni dello scritto di Catiforo (Cernovodeanu, 1975, pp. 86 - 90). Nel 1749 il volume I della versione neogreca fu tradotto dal grande boiardo valacco Matei Fărcăşanu. La sua versione, non stampata, si diffuse attraverso varie copie manoscritte. Sempre dal neogreco fu fatta in Moldavia la traduzione integrale dello scritto di Catiforo, dal chierico Cozma Vlahul del Metropolitanato di lasi, che si conservò sempre in copie manoscritte. Una situazione speciale è rappresentata da una versione manoscritta della Transilvania (Brasov), di cui non si conoscono le fonti utilizzate, la lingua fonte dalla quale fu eseguita la traduzione e, a nostro parere, il traduttore rumeno.

La presentazione del lavoro di Catiforo, delle fonti utilizzate, della traduzione in diverse lingue e il modo in cui si fecero le traduzioni è utile per illustrare il regime degli scritti nel Settecento, la circolazione dei libri e delle informazioni a livello europeo in questo periodo.

I. II primo volume di questa versione greca della Viaţa Marelui Petru è stato tradotto in rumeno in Valacchia, da Matei Fărcăşanu, nel 1749 (libri 1-4).

1. ms. miscel. 204 BAR, f. 99-241v, copia valacca di Lavrentie Dascălul, del 1749 (142 fogli, 21,5/15,5);

2. ms. miscel. 2353 BAR, f. 134-304, copia valacca di Rafail, monaco di Hurez, 1755 (170 fogli, 20/15);

3. ms. miscel. 2668 BAR, f. 8-175, copia valacca di Dumitru Rîmniceanu di Hurez, del 1767 (167 fogli, 22/16,5).

II. In Moldavia sono stati tradotti entrambi i volumi (6 libri), rimasti in 4 manoscritti, di cui 3 si conservano presso la BAR:

1. ms. 49 BAR, f. 2-211, Viaţa Marelui Petru, samoderjeţ a toată Rosia, copia moldava di Gavriil, Huşi, 1756 (209 file, 30/20,5); 
2. ms. 122 BAR, f. 2-212, 1765 (200 file, 31/21);

3. ms. 2581 BAR, f. 2-210, copia di Toader Meican, 1799, Moldavia (208 file, 30,5/22,5);

4. un altra copia si trova presso la Biblioteca „M. E. Saltîkov-Şcedrin” di San Pietroburgo, datata 1755.

III. Traduzione di Transilvania (Braşov)

1. ms. miscel. 3161 BAR, f. 1-186, Istoria ruşilor şi viaţa Marelui Petru, monarhul ruşilor, tradotto dal ministrante Rodion Popovici, Braşov, 1785, copiato nel 1788 da Zanfir Marco a Braşov (185 file, 20,5/16); f. 187-215 Istoria a marelui cneaz Dimitrie Ioanovici, samoderjiţul a toatei Rosiei;

2. ms. miscel. 2476 BAR, f. 4-144v, Viaţa lui Petru celui Mare, copia di Braşov; f. 73: "fevruarie 9, 1783, Ioan Zacionea, Sfîrşitul cărţii al doilea din" (140 file, 22/17); f. 320v : "1784, noiemvrie 2, Braşov, Ioan Zacionea".

I. Della vita e dell'attività di Matei Fărcăşanu si conoscono poche cose. Si sa che appartiene ad una grande familia nobile della Valacchia, con antenati noti sin dal Cinquecento per le loro gesta e per la loro contribuzione alla vita politica del paese. Verso l'inizio del Settecento, Matei Fărcăşanu è menzionato nei documenti come "logofăt", e nel 1746 come "biv vel şetrar". Era ancora in vita nel 1755, quando è attestato nei documenti come "vel serdar". Egli ebbe un figlio.Un altro Matei Fărcăşanu, attestato nei documenti della seconda metà del Settecento, è il figlio di questo Şerban e di Maria Cantacuzino ${ }^{1}$.

Informazioni riguardanti la formazione culturale di Matei Fărcăşanu si trovano soltanto nella parola al lettore che egli premette alla sua unica traduzione conosciuta, e cioè Viaţa Marelui Petru:

\begin{abstract}
"Essendo io coinvolto nei problemi e nelle vane preoccupazioni del passionale e molto rattristante mondo, ed essendo io ancora troppo poco avezzo allo studio della lingua greca, visto che nemmeno la grammatica ho imparato alla perfezione, se non con la poca conoscenza presa dalla pratica, notte e giorno come un ladro ho rubato qualche ora al sonno per completare questo libro perché le muse, ossia l'amore per la scienza che scandaglia nell'anima senza tormento, e mi è impossibile raccontare in dettaglio quanto io abbia sofferto fino a raggiungere questo traguardo che altro scopo non ha se non quello di essere utile al pubblico, dato che fino a oggi al nostro popolo sono mancati del tutto questi tipi di libri"?.
\end{abstract}

Non si è conservata la versione autografa del traduttore; il suo lavoro è noto tramite tre copie manoscritte effettuate in Valacchia nel Settecento.

Per il presente lavoro abbiamo studiato il ms. 2353 BAR, copiato nel 1755 dal monaco Rafail di Hurez. II monaco Rafail, dal nome laico Radu Bodeţ, era figlio di un certo prete Dumitru di Stînceşti-Prahova. Rimasto orfano, fu preso nel monastero di Hurez dove completò la sua educazione e dove si fece notare per la sua bella calligrafia e copiò più manoscritti che firmò Radu Copilul. Nel 1754, probabilmente al compimento dell'età maggiore, diventò monaco con il nome di Rafail ${ }^{3}$. Trascorse qualche tempo anche dal superiore Paisie Velicikovski di Dragomirna. Egli svolse un'intensa attività di revisore dei libri religiosi stampati a Rîmnic dai vescovi Chesarie e Filaret e continuò a copiare manoscriti dal contenuto teologico. Così contribuì alla stampa di un Octoih (1763), del Libro degli Salmi (1779) e di Minee, la cui edizione fu completa nel 1780, e copiò i manoscritti 2353, 5110, 2585, 2207, 2597, 2667, 3008, 2521, 2569, 2633, 2197 BAR (elencati in ordine cornologico) ${ }^{4}$.

Apprezzato per le sue qualità da alti fori ecclesiastici, fu nominato nel 1782 egumeno del monastero di Hurez. All'inizio del 1791, vecchio e malato, rinunciò alla carica, diventano ieromonaco, e nella primavera dello stesso anno morì a meno di 60 anni, lasciando al monastero un numero impressionante di libri e manoscritti. Dalla presentazione fatta al traduttore rumeno della Viaţa Marelui Petru, Matei Fărcăşanu, e da quella del copista Rafail risulta che entrambi erano valacchi di origine. I tratti linguistici del ms. 2353 BAR confermano il fatto che erano entrambi parlanti della varietà linguistica del sud (Valacchia).

Tratti specifici delle varietà del sud

Fonetica: cocoană 161v, cocon 200r; ciini 195r, mîni 154r, 202v, 280v, pîine 236v, 254r, 299r; ajutor 166r,

\footnotetext{
1 Per l'attività di Matei Fărcăşanu e cursuus honorum, si vedano Lecca, 1899, pp. 215-223 e Rădulescu, 1972, p. 453.

2 II testo rumeno del ms. 2353 BAR, f. 133v è: „Fiind împleticit întru neaşezările şi deşartele griji ale pătimaşei şi mult necăjitoarei lumi şi încă prea puţin fiind la învăţătura limbii elineşti, de vreme că nici gramatica desăvîrşit n-am învăţat, cu puţină cunoştinţă numai din praxis luînd, noaptea şi zioa ca un fur am furat cîte un ceas de somn ca să săvîrşesc această carte pentru că muzele, adică iubirea de învăţătură ce o şi caută de la suflet făr de turburare şi preste putinţă este a-ţ povesti cu amăruntul cît am răbdat pîn am cîştigat scoposul acesta carele la alt nu caută numai la cel de obşte folos, de vreme că pîn astăz neamul nostru să află lipsit de tot de acest feli de cărţi".

3 Per il nome di Rafail e dettagli sulla sua vita, si veda Dima, 1986-1987, pp.52-53.

4 Per la loro descrizione, si veda Ştrempel, 1978, pp. 23, 41 e 1983, pp. 321, 250.
} 
judecător 180v, mijloc 186r; genuche 214r, 249v, îngenuchia 214r

Morfologia: formele variabile ale articolului genitival: vicleşug al cazacilor 266r, împăraţi ai Moscovei 161v, colibi ale unor săraci 135r, luni ale verii 140v; identitatea formei de pers. 6 cu pers. 3 la prezent indicativ: vămile aduc [...] cîştig 212v.

Lessico: ginere „mire” 214r, zăpadă 140r, 153v, 222r, adverbul acolea 150v, 162v, 265r, 287v.

II testo che si trova nel ms. 2353 BAR presenta particolarità specifiche del rumeno letterario della metà del Settecento, caratterizzato dalla presenza di alcuni aspetti che hanno a che vedere con l'antica lingua letteraria, accanto a innovazioni più numerose nei settori della fonetica e del lessico.

I neologismi esistenti nel lavoro di Matei Fărcăşanu confermano la sua affermazione che era conoscitore soltanto della lingua greca. I prestiti dal turco, come caic 199r, calmuc 234v, conac 247v, farfurie 268v, sarai 192v erano conosciuti e avevano una grande circolazione nella lingua dell'epoca. La maggior parte dei neologismi sono provenieneti dal greco o sono di origine romanica, presi dall'originale greco. Tra i neologismi greci o romanici introdotti dalla lingua di partenza notiamo: amazoane 142r, amiral 238v şi amiraliu 204v, 215v, 239r, armadă 135r, 186r, 203v, 269r şi armată 171r, 245r, canţelar 291v, cardinal 138v, 156v, 227r, climă 139r, 141r, 283v, cliros 135v, 137v, colonel 253v, comedie $147 v$, deputaţi 202v, diamanturi 148v, 204v, dogmă 143v, 154r, eléctor 197v, 253r, 260r, evghenicesc 278v, 213r, familie $164 v, 172 r$, 193r, 299r, gheograf 298v, gramatică 132v, idolatri 137v, 298v, ighemon 288r, imperator 145v, 166r, 206r, madamă 204r, manifest 151r, 281v, 293r, monarh 134r, 195v, musici 206r, 290v, ofiţeri 163r, palat 144r, patos 136v, patrie 134r, 173r, 252r, 261v, pălărie 206r, politic 134v, pompă 149v, 163v, 226v, 292v, prinţip 161v, 166r, 295r, protimie 250r, psifos 210r, resedent 137r, 300r, rigă 207r, scop 214v, 232v şi scopos 132v, 134v, 167r, schimata 204v, tagma 158r, 162v, 205v, titlu 271r, tom 134r, viţăcolonel 277v.

Anche se alcuni neologismi conservano l'aspetto fonetico dell'etimo o hanno un valore morfologico specifico per la lingua letteraria antica, la lingua dello scritto di Fărcăşanu è leggera e offre al lettore, insieme alle informazioni contenute nel lavoro, anche una piacevole lettura. La semplicità e la scorrevolezza dello stile, determinate in grande misura dall'influsso dell'aspetto parlato della lingua rumena, portarono alla diffusione della traduzione di Fărcăşanu tramite copie manoscritte. Dal punto di vista del periodo quando fu scritta e delle particolarità linguistiche, Viaţa Marelui Petru collega la prima metà del Settecento, che è ancora segnata dalla cultura rumena antica, a quella della seconda metà del secolo che, per la lingua e la cultura rumena, rappresenta l'inizio della modernizzazione.

II. La versione moldava è stata anonima fino a quando se n'è occupato N.A. Ursu, che ne ha stabilito la paternità (Ursu, 2002, pp. 54-61). Supponendo che, durante gli otto anni trascrosi presso il Metropolitanato di lasi, il giovane ieromonaco Cozma Vlahul avesse fatto anche altre traduzioni, sia per il tipografo Duca Sotiriovici, sia per ordine del metropolita lacob Putneanul (1750-1760), lo studioso ha comparato la lingua di alcune traduzioni anonime elaborate all'epoca a lasi con la lingua della traduzione di Cîrja arhiereilor e ha costatato che lo ieromonaco Cozma aveva tradotto, sempre dal greco, Viaţa Marelui Petru, samoderjeţ a toată Rosia, rimasta in copie manoscritte, e dal slavo Alfavita sufletească, stampata nel 1755, e Sinopsis adecă adunare de multe învăţături, stampato nel 1757 . II testo della traduzione effettuata in Moldavia della Vieții Marelui Petru, samoderjeţ a toată Rosia si è conservato nei seguenti tre manoscritti: 49 BAR (datato 1756), 122 BAR (datato 1765) e 2581 BAR (datato 1799), nonché in un manoscritto della Biblioteca "M.E. Saltîkov-Şcedrin" di San Pietroburgo (datato 1755) (Cernovodeanu, 1975, pp. 86-90).

II traduttore in Moldavia della Viaţa Marelui Petru non è menzionato in nessuna delle quattro copie conosciute della traduzione. Dalla pagina-titolo della copia di San Pietroburgo risulta che la traduzione fu eseguita su iniziativa del metropolita lacob Putneanul.

N.A Ursu (2002) ha esaminato dal punto di vista linguistico e filologico i tratti della traduzione della Viaţa Marelui Petru contentnete un misto di particolarità linguistiche valacche e moldave, queste ultime dovute soprattutto ai copisti moldavi della traduzione. Lo studioso analizza attraverso metodi contrastivi le particolarità linguistiche comuni della Cîrja arhiereilor della Viaţa Marelui Petru e giunge alla conclusione che entrabmi i testi contengono elementi definitori per la lingua e lo stile dello ieromonaco Cozma Vlahul e che possono anch'esse essere invocate come prove del fatto che Viaţa Marelui Petru è stata tradotta dallo stesso Cozma.

III. A seguito di alcune note di copista, nella storia culturale rumena si è stabilita l'esistenza di una terza versione dello scritto di Catiforo, tradotta in Transilvania, a Braşov. In queste note si menziona che il testo è stato tradotto dal russo da Rodion Popovici: f. 1r del ms. 3161 BAR: Cartea politiei ruseşti şi vitejiile ruşilor, a monarhului Petru şi alţi împăraţi şi stăpînitori. Istoriile acestea sînt scoase de pe limba muscălească pe limba rumânească de domnia lui chirio chir Rodion Popovici, dascalul oraşului cetăţii Coronii... [copia del 1788 di Zamfir Marco, in Braşov]. A f. 187r si trova la nota seguente: Tălmăcitu-s-au această istorie din limba rusească pe limba rumânească de dumnealui chir dsascalul Radu Popovici în Braşov, la anno 1785, martie, 12. Şi s-au scris iar de... smeritul Zanfir Marco, în Braşov, la anno 1788, 
april 29 .

In effetti, verso la metà del Settecento c'è stata anche una versione in russo dei due volumi di Catiforo con titolo Zitie Petra Velikago, Imperatora i Samoderzca Vserossijskago, otca otecestva, traduzione di Stefan Ivanovici Pisarev et all., San Pietroburgo, 1772.

Investigando il ms. $3161 \mathrm{BAR}$, siamo giunti alle seguenti conclusioni. II manoscritto è un miscellaneo che comprende: Viaţa Marelui Petru (ff. 2r-186v), Istoria a marelui cneaz Dimitrie loanovici, samoderiiţul a toatei Rosiei (ff. 187-215) e Începerea şi istoria războiului de la cetatea Beciului (ff. 216r-279v), mentre la fine manca.

Il primo testo è una traduzione parziale dello scritto di Catiforo, Vita di Pietro, e comprende i libri 1-4 del tomo I. Nel manoscritto segue un altro frammento, intitolato libro 5, ma il quale, anche se viene menzionato come appartenente allo stesso lavoro, è completamente diverso dal testo di Catiforo e contiene l'interpolazione di un inventario delle truppe svedesi e russe dopo la guerra tra la Russia e la Svezzia e della cronaca di Nicolae Costin sul il periodo 1709-1711 (Dima, 2012, pp. 75-86) $)^{5}$.

Percorrendo i primi quattro libri della traduzione del testo di Catiforo del ms. miscel. 3161 BAR, abbiamo costatato che nel testo non si ritrovano lessemi presi in prestito dal russo, come sarebbe stato naturale nella relazione tra la lingua di partenza e la lingua d'arrivo, ma numerose parole che hanno o l'origine nel greco o forme e pronunce caratteristiche per la lingua greca. Nella cultura rumena, l'influsso greco era forte, soprattutto a livello ufficiale, politico e amministrativo, ma anche culturale, potendosi parlare di un bilinguismo greco-rumeno nel periodo fanariota. Gli esempi che faremo non fanno parte delle parole di origine greca frequenti nell'epoca, ma rappresentano forme rare per le quali bisogna trovare una spiegazione. Per questo abbiamo intrapreso una comparazione linguistica e testologica tra la versione italiana del 1748, disponibile online, quella greca di Kanghellarios, tomo $I^{6}$, un numero di pagine della versione russa, che si trova presso la biblioteca di Berlino ${ }^{7}$, e le versioni rumene.

Utilizzeremo il metodo contrastivo e le abbreviazioni: it. per la forma presente nel testo italiano, ngr. per la forma della versione greca, rus. per la forma della traduzione russa, Val. (ms. 2353 BAR) per la forma della versione di Matei Fărcăşanu, Mold. per la forma della versione di Cozma Vlahul (ms. 49 BAR) e Trans. per la forma della versione transilvana, copiata a Braşov (ms. 3161 BAR), facendo in seguito alcuni esempi da questi testi:

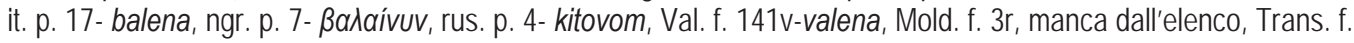
4r- valena;

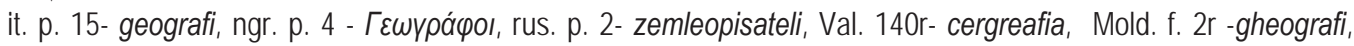
Trans. f. $2 r$-gheografii;

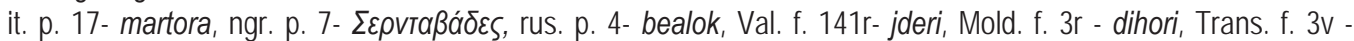
zerdavale;

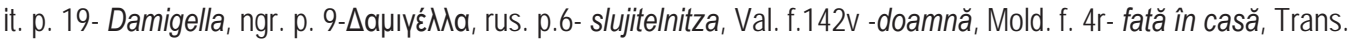
f. 4v- Damighella; Secheliei;

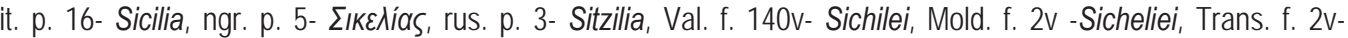

it. p. 17-Thubal, ngr. p. 7-Ooßśd, rus. p. 5- Thovelem, Val. f. 141r- Thovel, Mold. f. 3v-Thovel, Trans. f. 3v- Tovel;

it. p. 18- Rurich, ngr. p. 8- Poupıxos, rus. p. 5- Riuric, Val. f. 142r- Rurih, Mold. f. 3v- Rurih, Trans. f.4r-Ruritos.

A pagina 19 nel testo italiano si ritrova un paragrafo in cui si parla delle memorie di Nestesouran, il compilatore di una vita dello zar Pietro stampata in Olanda, e dove si fanno alcune considerazioni riguardanti la conversione di Vladimir al protestantismo. II frammento manca nella traduzione russa, essendone eliminato (si veda p. 7), ma si ritrova nella versione della Transilvania (f. 5r), della Valacchia (ff. 143r - 143v) e della Moldavia (f. 4v).

Attraverso gli esempi dati, in cui abbiamo presentato alcuni lessemi che nominano animali, funzioni, antroponimi, toponimi, abbiamo mostrato le differenze testologiche tra le varie versioni e possiamo trarne le seguenti conclusioni:

1. La versione della Transilvania non ha avuto come lingua di partenza il russo, come si afferma all'inizio del testo, ma il greco. II fatto che sul frontispizio si menziona che il testo sia stato tradotto dal russo è sicuramente un errore del copista, la confusione essendo dovuta probabilmente al fatto che nel manoscritto miscellaneo si trovano anche altri due testi che riguardano la storia della Russia, il che ha portato all'idea che tutti i testi fossero tradotti dal russo.

2. Le tre versioni rumene hanno come fonte la traduzione greca di Kanghellarios.

\footnotetext{
${ }^{5}$ Abbiamo descritto il contenuto del libro 5 e la paternità del testo interpolato.

${ }^{6}$ Abbiamo ottentuo una copia dalla biblioteca dell'Università Aristoteles di Salonicco, grazie all'amabilità della direzione della biblioteca. ${ }^{7}$ La copia è stata ottenuta per la gentilezza del prof. Andrei Hoisie, del drd. Markus Winkler e della bibliotecaria Camelia Boca, ai quali esprimiamo anche in questa occasione la nostra gratitudine.
} 
3. Dalla investigazione del testo russo e dagli esempi presentati si osservano alcune forme che provengono dal greco 0 alcune pronunce influenzate dal greco, più precisamente dalla traduzione di Kanghellarios, dunque la traduzione russa è stata fatta dall'intermediario greco e non direttamete dal testo italiano di Catiforo.

4. Dato che il protografo non si è conservato, i manoscritti citati rappresentano copie di date non concordi e le informazioni sul traduttore della versione transilvana sono confuse, questa rimane ancora nella nostra attenzione per stabilirne la paternità.

\section{Acknowledgement}

II presente lavoro è realizzato nell'ambito del progetto di ricerca PN-II-CT-ERC-2012-1 - 5 ERC, The Impulse of the Enlightenment in some Romanian Translations in the 18th Century, finanziato dal CNCS, per il periodo giugno 2012giungo 2014

\section{References}

Cernovodeanu, P. (1975). Préoccupations en matière d'histoire universelle dans l'historiographie roumaine aux XVII-e et XVIII-e siècles. Revue des études sud-est européennes, XII, 1975, nr. 1, p. 86-90.

Dima, E. (1986-1987). Primele traduceri din Halima în limba română, Anuar de lingvistică și istorie literară, XXXI, A (pp. 39-64). lasi: Univ. "Al. I. Cuza".

Dima, E. (2012). Una versione inedita della cronaca della Moldavia dal 1709 al 1711 di Nicolae Costin, interpolata nella "Viaţa lui Petru. Analele știintịifice ale Universității "Alexandru loan Cuza" din lasi, IIle, Lingvistica, LVIII (pp. 75-86). Iași: Univ. "Al. I. Cuza".

George Lecca, G. (1899). Familiile boiereşti române, Bucureşti, 1899.

Kechagioglu, G. (1994). Traduzioni neogreche del XVIII secolo: l'italiano come lingua veicolare. Testi letterari italiani tradotti in greco (dal '500 ad oggi), a cura di M. Vitti, Soveria Mannelli. Soveria Mannelli: Rubbettino.

Losacco, M. (2001). Antonio Catoforo e Giovanni Veludo interpreti di Fozio. Bari: Dedalo.

Rădulescu, Theodora (1972). Sfatul domnesc şi alţi mari dregători ai Ţării Româneşti din secolul al XVIII-lea. Revista Arhivelor, an 1972 , vol. XXXIV, nr. 3.

Ştrempel, G. (1978) Catalogul manuscriselor româneşti. B.A.R., 1-1600, I. Bucureşti: Academiei

Ştrempel, G. (1983) Catalogul manuscriselor româneşti. B.A.R. 1601-3100, II. Bucureşti: Academiei.

Ursu, N.A. (2002). Contribuții la istoria culturii românești. Studii și note filologice (pp. 54-61). lasi: Cronica. 